\title{
DISTRIBUCIÓN DE LAS LEGUMINOSAS DE LA PARTE ALTA DE LA CUENCA LA GALLEGA. MORROPÓN. PIURA.
}

Aldo Ceroni Stuva ${ }^{1}$.

\section{Resumen}

Se estudió la distribución de las leguminosas de la parte alta de la cuenca La Gallega (Morropón, Piura). Las plantas fueron recolectadas entre los 1,350 y 3,100 msnm. Se registraron los rangos altitudinales de las especies. Las 21 especies colectadas pertenecen a 3 subfamilias y 9 tribus. La subfamilia Papilionoideae y la tribu Phaseoleae presentaron una mayor distribución. La especie con menor distribución fue Caesalpinia spinosa, encontrada sólo en los alrededores de Santo Domingo, mientras que las especies más ampliamente distribuidas fueron Erythrina edulis y Vigna luteola. También se encontraron 10 nuevos registros de distribución de leguminosas para el Perú.

Palabras clave: Distribución, rangos altitudinales, familia, subfamilia, tribu.

\begin{abstract}
The leguminous plants distribution in the highest part of the La Gallega basin (Morropón, Piura) was studied. The plants were collected between 1,350 and 3,100 masl. Altitudinal ranges of the species were registered. The 21 collected species belong to 3 subfamilies and 9 tribes. The greatest distribution corresponded to the Papilionoideae subfamily and to the Phaseoleae tribe. Caesalpinia spinosa was the species with the narrowest distribution and was found only near Santo Domingo, while species such as Erythrina edulis and Vigna luteola were those of the widest distribution. We also found 10 new records of leguminous plants distribution in Perú.
\end{abstract}

Key words: Distribution, altitudinal ranges, family, subfamily, tribe.

\section{Introducción}

Una de las familias botánicas más importantes en la flora tropical es el de las Leguminosae o Fabaceae. Esta familia incluye numerosas especies con una gran variedad de portes y formas de vida. Muchas de sus especies tienen una enorme importancia económica para el hombre (Heywood, 1985). En muchos países en desarrollo, las leguminosas alimenticias constituyen la principal fuente de proteína en su alimentación (Bruno, 1990). Un estudio de distribución de las especies de esta familia es muy importante por cuanto permite por un lado reforzar la situación taxonómica de las especies y por otro lado, conocer la ubicación de recursos vegetales tanto para el uso directo, industrial, así como, fuente de recursos fitogenéticos. Estudios sobre leguminosas en el Perú se hallan en forma fragmentaria en diversas obras a nivel nacional e internacional, tales como los de MacBride (1943), Gómez (1960), Arce (1970), Carrillo (1974), Cerrate (1979), Ferreyra (1987), Barneby (1988), Tovar (1990), Brako \& Zarucchi (1993), Flores (1997) y Reynel \& Pennington (1997), todos ellos en estudios florísticos y revisión de géneros (Flores, 1997; Ceroni, 1998). Las leguminosas son también una de las familias con mayor número de especies para el norte peruano. Además, por ser los Andes del norte más bajos en altitud, debido al Abra de Porculla o Deflexión de Huancabamba, es una región ampliamente reconocida como una fuente de diversidad primaria y endemismo en muchos grupos de plantas (Sagástegui et al., 1999). El presente trabajo tuvo como objetivo aportar al conocimiento de la distribución de las leguminosas de la parte alta de la cuenca La Gallega, para lo cual se registró el rango altitudinal de las especies, en cada una de las localidades. El estudio ha sido centrado en la parte alta por haberse observado que esta zona presenta una vegetación más rica en especies, muchas de las cuales son utilizadas por los pobladores.

\section{Materiales y métodos}

\section{Ubicación}

La cuenca La Gallega está ubicada al suroeste de la microregión andina central, en el Departamento de Piura, dentro de la gran Cuenca del Piura. La microregión andina central se ubica entre los $4^{\circ} 90^{\prime} \mathrm{y}$ $5^{\circ} 10^{\prime} \mathrm{LS}$ y entre los $79^{\circ} 30^{\prime}$ y $80^{\circ} 10^{\prime}$ LO (Figura 1 ).

\section{Caracterización de la zona de estudio Área}

La cuenca La Gallega está formada por el río La Gallega que nace en el lado sur de los Altos, en el cerro Peña de Huaycas. Su longitud es de $37 \mathrm{Km}$. Tiene una superficie de 17,897 Ha. y un área de 185. $60 \mathrm{Km}^{2}$ (CIGA, 1990). La parte alta es aquella comprendida entre Chungayo y Los Altos, en la divisoria de aguas, es decir entre 1,350 y 3,100 msnm.

\footnotetext{
${ }^{1}$ Departamento Académico de Biología. Universidad Nacional Agraria La Molina. Av. La Molina s/n Lima Perú. Apartado postal 456. Lima 100 Perú. Fax: 3496015. Correo electrónico: aceroni@lamolina.edu.pe.
} 


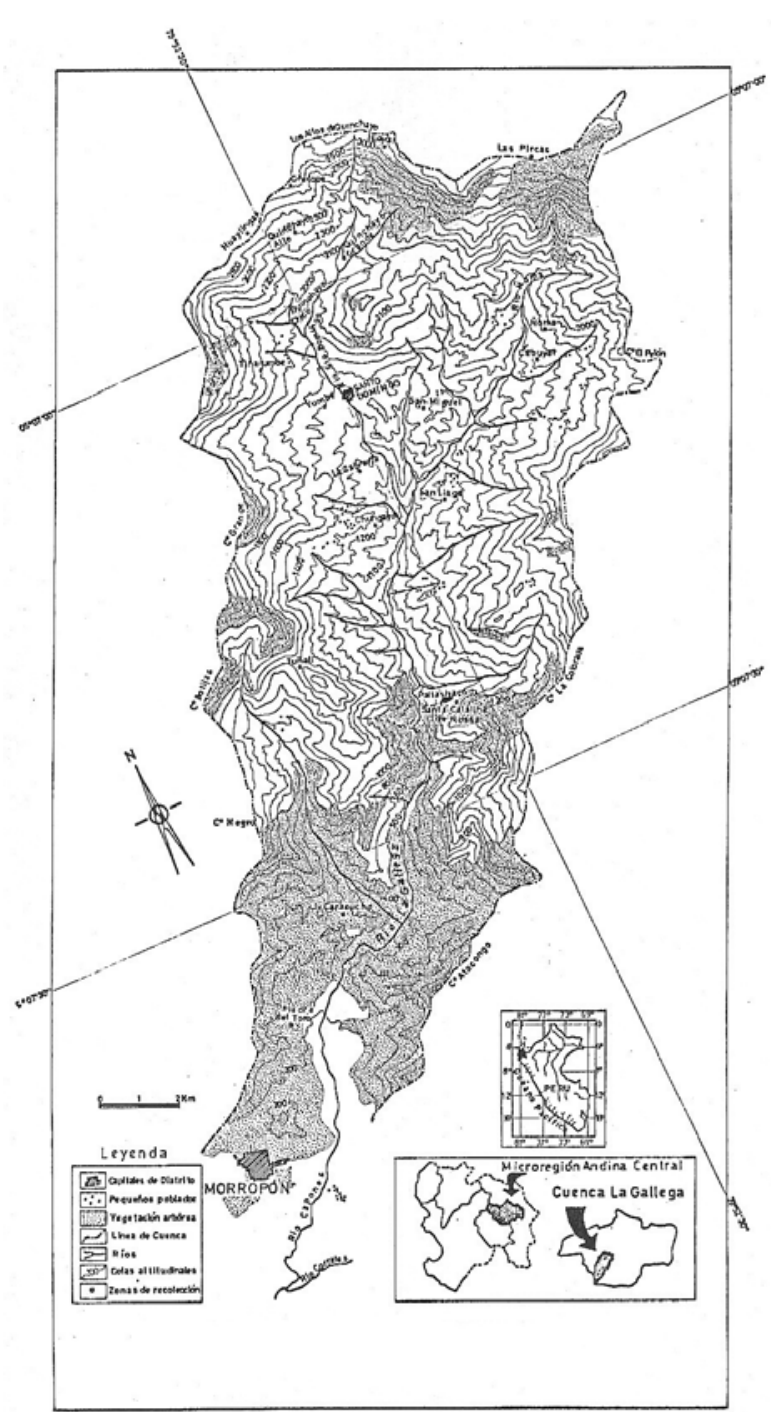

Figura 1. Cuenca La Gallega. Santo Domingo. Morropón. Piura.

\section{Clima}

La parte alta es húmeda con precipitaciones de 800 a 1,000 mm/año. La humedad ambiental también hace notar sus diferencias entre la parte baja, media y alta de la cuenca. Esta última es muy húmeda y con nieblas casi estables durante el verano.

\section{Suelos}

La parte alta presenta suelos arcillosos y pedregosos. A la altura de Santo Domingo, los suelos son arcillosos-limosos y de color pardo.

\section{Fisiografía}

La cuenca está formada por numerosas quebradas que han excavado profundamente sus lechos. El ancho máximo del valle es de $11 \mathrm{Km}$. en su sector alto y el mínimo de $6 \mathrm{Km}$. en su sector bajo. En el fondo del valle hay terrazas escalonadas con un ancho medio de $1 \mathrm{Km}$. A la altura de Santo Domingo se abre una repisa y el relieve aquí tiene una pendiente de $10 \%$ al $15 \%$.

\section{Ecología}

De acuerdo al Mapa Ecológico del Perú (INRENA, 1995) la parte alta de la cuenca La Gallega se encuentra comprendida entre 3 zonas de vida: bosque seco-Montano Bajo Tropical (bs-MBT), bosque húmedo-Montano Bajo Tropical (bh-MBT) y bosque muy húmedo-Montano Tropical (bmh-MT).

\section{Metodología}

Se realizaron viajes de recolección de las especies botánicas, registrándose las altitudes, así como la especie o especies dominantes en cada lugar. Los viajes de recolección se realizaron durante 1991, 1994 y 1995 en los meses de abril, agosto, octubre y noviembre. Las plantas fueron recolectadas entre los 1,350 y 3,100 msnm., en los siguientes lugares: Santo Domingo, Ñoma, Yumbe, Portachuelo, Chungayo, Cabuyal, Tiñarumbe, Chicope, Quinchado Chico, Quinchado Grande, Quinchado Alto y Las Pircas. El material botánico fue posteriormente determinado en el Herbario Weberbauer del Departamento Académico de Biología de la Universidad Nacional Agraria La Molina (MOL) y en el Herbario del Museo de Historia Natural de la Universidad Nacional Mayor de San Marcos (USM).

\section{Resultados y discusión Especies colectadas}

Las leguminosas colectadas en la parte alta de la cuenca La Gallega fueron un total de 21, pertenecientes a las tres subfamilias: 1 Mimosoideae; 1 Caesalpinioideae y 19 Papilionoideae (Tabla 1). Asimismo, las especies de la última subfamilia pertenecen a 9 tribus (Tabla 2).

\section{Distribución de las especies}

Las especies de leguminosas encontradas en el área de estudio se distribuyen en su mayoría de manera amplia. Sin embargo, a fin de conocer mejor esta distribución, se destaca la ubicación de los diferentes lugares de colección para cada una de ellas. Así la Mimosoideae Inga vera Willdenow, se encontró en las partes bajas y medias del área de estudio, en Santo Domingo y Ñoma, entre los 1,500 y 1,800 msnm. La Caesalpinioideae Caesalpinia spinosa (Molina) Kuntze, se encontró sólo en los alrededores de Santo Domingo a $1,500 \mathrm{msnm}$, la cual aparentemente tendría una distribución más restringida (Figura 2). Las Papilionoideae presentaron una mayor distribución en toda el área de estudio desde Chungayo hasta las Pircas, cubriendo un rango altitudinal entre 1,350 y 3,100 msnm. (Figuras 2, 3 y 4). Entre ellas, la tribu Phaseoleae fue la de mayor distribución ya que sus representantes fueron encontrados en la mayoría de los puntos de colección: Chungayo, Yumbe, Santo Domingo, Tiñarumbe, Cabuyal, Noma, Quinchayo Chico y Quinchayo Grande, 
cubriendo un rango altitudinal entre 1,350 y 1,900 msnm (Figura 4). En cuanto a las especies mismas, las que presentaron mayor distribución, considerando el número puntos de colección y la distancia entre los puntos, fueron: Amicia glandulosa H.B.K., Dalea coerulea (L.f.) Schinz \& Thellung, Desmodium campyloclados Hemsley, Erythrina edulis Triana ex Micheli, Fiebrigiella gracilis Harás., Inga vera Willdenow, Lupinus mutabilis Sweet, Otholobium holosericeum (Barneby) Grimes, Phaseolus polyanthus Greenman y Vigna luteola (Jacquin) Bentham; siendo las más ampliamente distribuidas Erythrina edulis Triana ex Micheli y Vigna luteola (Jacquin) Bentham.

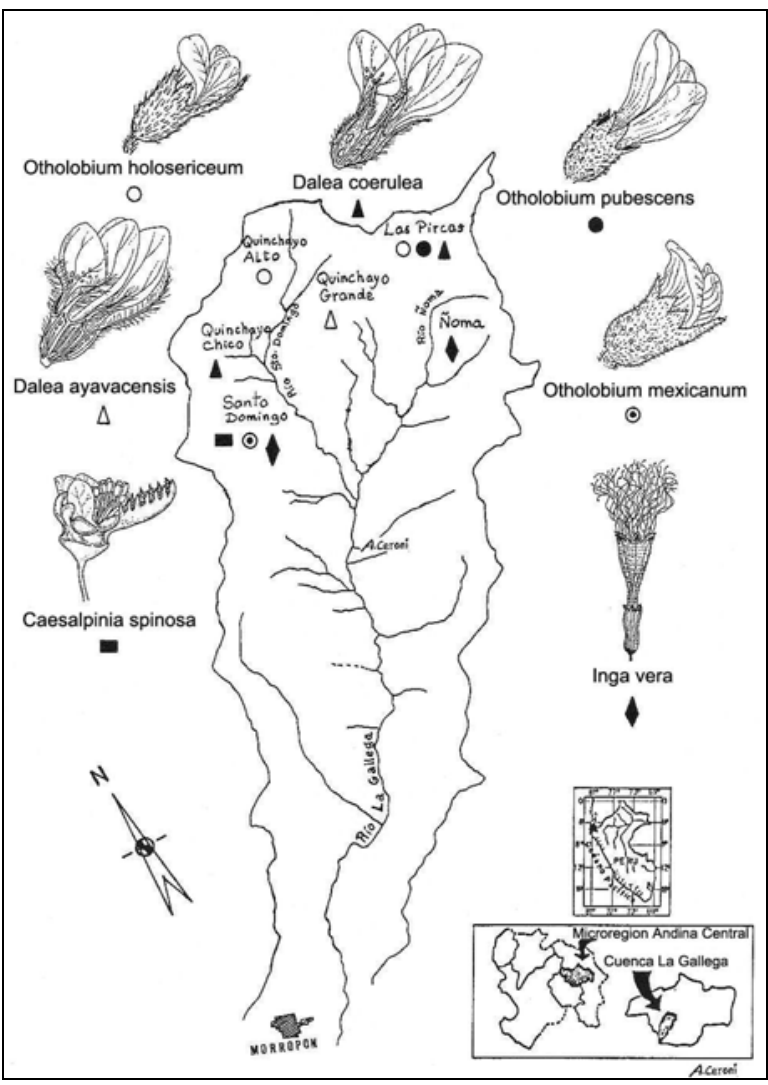

Figura 2. Lugares de colección de la Mimosoideae, Caesalpinoideae y las Papilionoideae de las Tribus Psoraleeae y Amorpheae en la parte alta de la cuenca La Gallega. Morropón. Piura.

Al hacer una revisión de la distribución en el Perú de las especies encontradas aquí, basada principalmente en la información dada por Brako \& Zarucchi (1993) y la obtenida de los herbarios USM y MOL, se encuentra que varias especies no están reportadas para Piura o para la zona específica de Santo Domingo en Morropón. Por lo tanto especies como Crotalaria sagittalis L., Clitoria pozuzoensis Macbride, Dalea coerulea (L.f.) Schinz \& Thellung, Desmodium molliculum (H.B.K.) DC., Inga vera Willdenow, Medicago polymorpha L., Otholobium holosericeum (Barneby) Grimes, Phaseolus polyanthus Greenman, Vicia andicola H.B.K. y Vigna luteola (Jacquin) Bentham, serían casos de nuevos reportes de distribución para el Perú (Ceroni, 1998). Entre ellas, por ejemplo, al consultar con uno de los autores del estudio del Género Inga en el Perú (Reynel \& Pennington, 1997) para la respectiva determinación, fue interesante conocer que la especie Inga vera Willdenow, también llega hasta Piura.

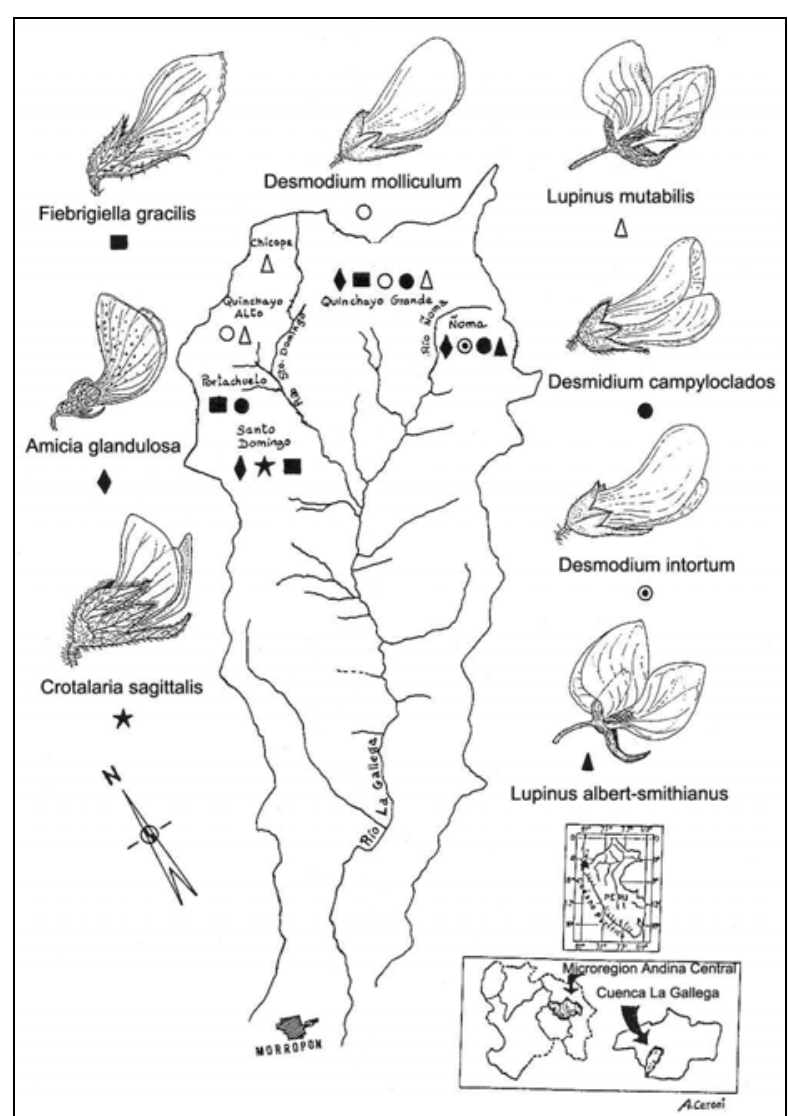

Figura 3. Lugares de colección de las Papilionoideae de las Tribus Desmodieae, Aeschynomeneae, Crotalarieae y Genisteae en la parte alta de la cuenca La Gallega. Morropón. Piura.

\section{Conclusiones}

1. Se registraron un total de 21 especies de leguminosas, pertenecientes a 3 subfamilias y 9 tribus. 2. La subfamilia Papilionoideae presenta una mayor distribución desde Chungayo hasta las Pircas, cubriendo un rango altitudinal entre 1,350 y 3,100 msnm.

3. La tribu Phaseoleae presenta una mayor distribución, con representantes encontrados en la mayoría de puntos de colección, en un rango altitudinal entre 1,350 y $1,900 \mathrm{msnm}$.

4. La especie con menor distribución fue Caesalpinia spinosa, encontrada sólo en los alrededores de Santo Domingo, mientras que las especies más ampliamente distribuidas fueron Erythrina edulis y Vigna luteola. 
Diciembre 2003

5. Las especies: Crotalaria sagittalis, Clitoria pozuzoensis, Dalea coerulea, Desmodium molliculum, Inga vera, Medicago polymorpha, Otholobium holosericeum, Phaseolus polyanthus, Vicia andicola y Vigna luteola, constituyen nuevos registros de distribución para el Perú.

6. Las localidades con mayor concentración de leguminosas fueron Santo Domingo, Noma y Quinchayo Grande, a 1,475, 1,640 y 1,970 msnm, respectivamente.

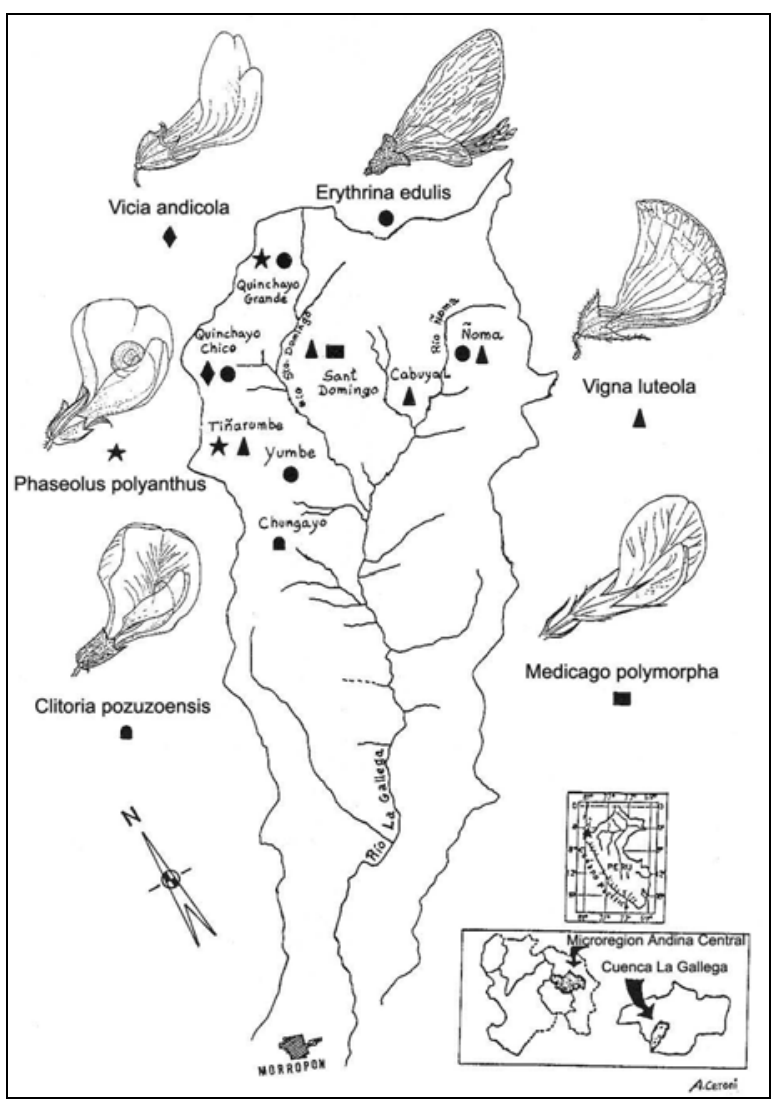

Figura 4. Lugares de colección de las Papilionoideae de las Tribus Vicieae, Trifolieae y Phaseoleae en la parte alta de la cuenca La Gallega. Morropón. Piura.

\section{Agradecimientos}

El autor expresa su agradecimiento al M.Sc. Juan Torres por sugerir el área de estudio y apoyar en las primeras visitas de reconocimiento, a la Bach. Alina Pace, la Blga. Susana Gushiken y el Blgo. Omar Carrión por su apoyo en las salidas de campo, a la Ing. Luz María Jiménez, por los contactos necesarios para contar con guías durante los recorridos en el área de estudio, a la Dra. Elida Carrillo, Dra. Magda Chango, Dra. Graciela Vilcapoma, M.Sc. Mercedes Flores y al Dr. Carlos Reynel por las facilidades dadas en los Herbarios MOL y UNMSM, y a la Dra. Elsa Fun por el apoyo financiero y logístico de la Central Peruana de Servicios (CEPESER) de Piura. Sin todos ellos hubiera sido difícil poder realizar el presente estudio.
Tabla 1. Lista de leguminosas colectadas en la parte alta de la cuenca La Gallega. Santo Domingo. Morropón. Piura.

\begin{tabular}{|c|c|}
\hline $\begin{array}{l}\text { SUBFAMILIA / NOMBRE } \\
\text { CIENTÍFICO }\end{array}$ & $\begin{array}{l}\text { NOMBRE } \\
\text { COMÚN }\end{array}$ \\
\hline $\begin{array}{l}\text { MIMOSOIDEAE } \\
\text { Inga vera Willdenow }\end{array}$ & guaba de zorra \\
\hline $\begin{array}{l}\text { CAESALPINIOIDEAE } \\
\text { Caesalpinia spinosa (Molina) Kuntze }\end{array}$ & taya \\
\hline 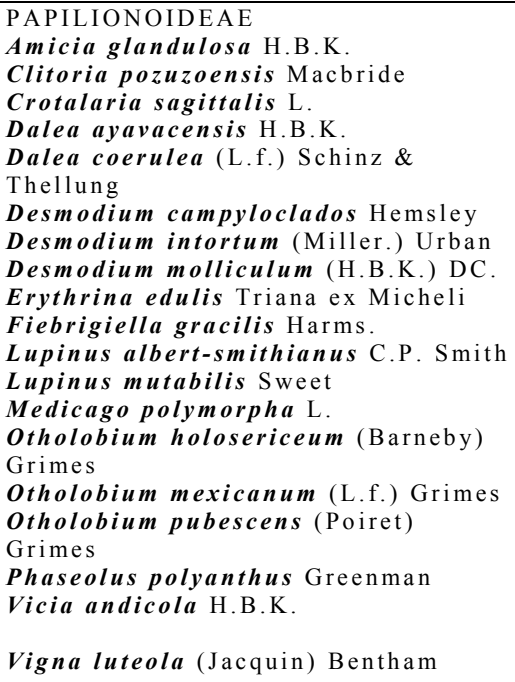 & $\begin{array}{c}\text { manayupa } \\
\text { pajul } \\
\text { tarwi } \\
\text { talalo } \\
\text { carretilla } \\
\text { pulén de vaca } \\
\\
\text { poleo grande } \\
\text { pulén de } \\
\text { tomar } \\
\text { frijolde vida } \\
\text { arvejita de } \\
\text { puga } \\
\text { raíz pelada }\end{array}$ \\
\hline
\end{tabular}

Tabla 2. Tribus de las Papilionoideae colectadas en la parte alta de la cuenca La Gallega. Morropón. Piura.

\begin{tabular}{|c|c|}
\hline TRIBU & ESPECIE \\
\hline DESMODIEAE & $\begin{array}{l}\text { Desmodium campyloclados } \\
\text { Hemsley } \\
\text { Desmodium intortum (Miller.) } \\
\text { Urban } \\
\text { Desmodium molliculum (H.B.K.) } \\
\text { DC. }\end{array}$ \\
\hline AESCHYNOMENEAE & $\begin{array}{l}\text { Amicia glandulosa H. В.К. } \\
\text { Fiebrigiella gracilis Harms. }\end{array}$ \\
\hline PSORALEEAE & $\begin{array}{l}\text { Otholobium holosericeum } \\
\text { (Barneby) Grimes } \\
\text { Otholobium mexicanum (L.f.) } \\
\text { Grimes } \\
\text { Otholobium pubescens (Poiret) } \\
\text { Grimes }\end{array}$ \\
\hline AMORPHEAE & $\begin{array}{l}\text { Dalea ayavacensis H.B.K. } \\
\text { Dalea coerulea (L.f.) Schinz \& } \\
\text { Thellung }\end{array}$ \\
\hline CROTALARIEAE & Crotalaria sagittalis $\mathrm{L}$. \\
\hline GENISTEAE & $\begin{array}{l}\text { Lupinus albert-smithianus C.P. } \\
\text { Smith } \\
\text { Lupinus mutabilis Sweet }\end{array}$ \\
\hline VICIEAE & Vicia andicola H.В.К. \\
\hline TRIFOLIEAE & Medicago polymorpha $\mathrm{L}$. \\
\hline PHASEOLEAE & $\begin{array}{l}\text { Clitoria pozuzoensis Macbride } \\
\text { Erythrina edulis Triana ex } \\
\text { Micheli } \\
\text { Phaseolus polyanthus Greenman } \\
\text { Vigna luteola (Jacquin) Bentham }\end{array}$ \\
\hline
\end{tabular}




\section{Literatura citada}

Brako L. \& Zarucchi J. 1993. Catalogue of the Flowering Plants and Gymnosperms of Perú. Monograhs in Systematic Botany from the Missouri Botanical Garden. Vol 45.

Bruno H.J. 1990. Leguminosas Alimenticias. Distribuidora "Faede". S.A. Lima. Perú.

Ceroni A. 1998. Estudio Taxonómico de la Familia Leguminosae de la Cuenca La Gallega. Santo Domingo. Parte Alta (Morropón, Piura). Tesis para optar el Grado de Magíster en Botánica Tropical. Universidad Nacional Mayor de San Marcos.

CIGA. 1990. Cuaderno de Geografía Aplicada. Proyecto de Desarrollo Rural Integral de la Sierra Central del Departamento de Piura. PUCORSTOM. Primera parte: El Medio Natural.

Flores M. 1997. La Familia Leguminosae en el Valle del Chillón - parte media y alta. Tesis para optar el Grado de Magíster en Botánica Tropical.
Universidad Nacional Mayor de San Marcos.

Heywood V.H. 1985. Las Plantas con Flores. Editorial Reverté S.A. España. : 145-148.

INSTITUTO NACIONAL DE RECURSOS NATURALES (INRENA). 1995. Mapa Ecológico del Perú. Guía explicativa. Lima-Perú.

MacBride J.F. 1936 - 1971. Flora of Perú. Botanical Series. Field Museum of Natural History. U.S.A.

Sagástegui A., Dillon M., Sánchez I., Leiva S. \& Lezama P. 1999. Diversidad Florística del Norte de Perú. Tomo I. World Wildlife Fund Inc. UPAO.

Soukup J. 1987. Vocabulario de los Nombres Vulgares de la Flora Peruana y Catálogo de los Géneros. Editorial Salesiana. Lima. Perú.

Weberbauer A. 1945. El Mundo Vegetal de los Andes Peruanos. Estudio Fitogeográfico. Estación Experimental Agrícola de La Molina. Ministerio de Agricultura. Lima.: 6, 30, 31. 